ISSN 0001-6012/2019/61/2/73-76 Acta Médica Costarricense, ( 2019 Colegio de Médicos y Cirujanos de Costa Rica

\title{
Opinión
}

\section{Usos, contexto regulatorio y ético de la talidomida en Costa Rica}

\author{
(Thalidomide in Costa Rica: uses, regulatory and ethical context)
}

Ana Rivera-Chavarría ${ }^{1}$, Azálea Espinoza-Aguirre ${ }^{2}$

\section{Resumen}

En las últimas décadas, el descubrimiento de las propiedades antinflamatorias, antiangiogénicas e inmunorreguladoras de la talidomida, ha propiciado su utilización como terapia alternativa o de segunda elección para diversas patologías. El medicamento forma parte de la Lista Oficial de Medicamentos y Normativa de la Caja Costarricense de Seguro Social. El resurgimiento del medicamento y sus análogos, los cambios en el comportamiento reproductivo de la población, avances en las técnicas de reproducción asistida, investigación biomédica y la judicialización de la medicina, hacen necesaria una revisión de sus indicaciones actuales, y medidas de seguridad para prevenir el efecto adverso más grave: la teratogénesis. El objetivo de esta investigación fue presentar los resultados de una revisión bibliográfica relacionada con los usos actuales, así como el contexto regulatorio y ético de la talidomida en Costa Rica.

Descriptores: talidomida, IMiDS, aspectos éticos, regulación.

\section{Abstract}

In recent decades, thalidomide's anti-inflammatory, anti-angiogenic and immunoregulatory

Afiliación de los autores: 'Unidad Salud y Nutrición, Instituto Costarricense de Investigación y Enseñanza en Nutrición y Salud (INCIENSA). 2Dirección de Vigilancia de la Salud, Ministerio de Salud de Costa Rica.

Fuentes de apoyo: esta investigación fue posible gracias al apoyo financiero del Instituto Costarricense de Investigación y Enseñanza en Salud y Nutrición (Inciensa), Tres Ríos, Cartago, Costa Rica y Dirección de Vigilancia de la Salud Ministerio de Salud de Costa Rica.

Conflictos de interés: las autoras no tienen ningún conflicto de interés.

Abreviaturas: Cereblon, CRBN Caja Costarricense de Seguro Social, CCSS; Lista Oficial de Medicamentos de Seguro Social, LOM; Mieloma Múltiple, MM Food and Drug Administration, FDA.

凶arivera@inciensa.sa.cr

properties have led as an alternative or second-choice therapy for various pathologies. It is currently included in the Official List of Medicines and Regulations of the Costa Rican Social Security (LOM). However, thalidomide's resurgence and its analogues, reproductive behavior changes, assisted reproduction technology, biomedical research and medical judicialization, indicates that a review of the current indications and safety measures are necessary to prevent the most serious adverse effect: teratogenesis. In this research, we aim to summarize thalidomide's current uses, regulatory and ethical context in Costa Rica.

Keywords: Thalidomide, IMiDS, ethical issues, regulation.

Fecha recibido: 17 de diciembre 2018

Fecha aprobado: 28 de febrero 2019

La talidomida es un fármaco no barbitúrico (derivado sintético del ácido glutámico), con actividad sedante y antiemética, introducido en los años cincuenta en Alemania y comercializado en el Reino Unido y otros países a partir de 1958 como sedante, por su rápida acción y "aparente seguridad" ${ }^{1,2}$

Poco después de su introducción en el mercado se registraron, en todo el mundo, dismelias, malformaciones congénitas en los miembros, tales como amelia, focomelia, y ausencia del pulgar o los dedos, entre otros, por 10 general extremadamente infrecuentes. ${ }^{3}$ No obstante, no fue hasta principios de los años setenta cuando se eliminó su uso de todo el mundo., ${ }^{4,5}$ Tras su retiro del mercado, continuó la posibilidad de administrar el fármaco como sedante e hipnótico, exclusivamente para pacientes con enfermedad de Hansen en los Estados Unidos.

En las últimas décadas, el descubrimiento de sus propiedades antinflamatorias, 
antiangiogénicas e inmunorreguladoras, ha propiciado su utilización como terapia alternativa o de segunda elección en la sarcoidosis, la úlcera aftosa en pacientes con virus de la inmunodeficiencia humana, la estomatitis aftosa recurrente, la enfermedad de Behçet, el prurigo nodular y el prurigo actínico, en la enfermedad de injerto contra huésped crónica, en leucemias agudas y síndromes mielodisplásicos.,

La talidomida está incluida en la Lista Oficial de Medicamentos y Normativa de la Caja Costarricense de Seguro Social (LOM). ${ }^{8}$ Dado el resurgimiento del medicamento y sus análogos, cambios en el comportamiento en la población en edad reproductiva, avances en las técnicas de reproducción asistida, investigación biomédica y la judicialización de la medicina, es necesaria una revisión de sus indicaciones actuales y medidas de seguridad, para prevenir el efecto adverso más grave: la teratogénesis.

El objetivo de este trabajo fue presentar los resultados de una revisión bibliográfica relacionada con los usos, así como el contexto regulatorio y ético de la talidomida, en Costa Rica.

\section{Mecanismos de acción de la talidomida}

Los mecanismos de acción de los efectos de la talidomida no están del todo esclarecidos. ${ }^{4,9,10}$ La bibliografía menciona que la talidomida posee propiedades inmuno-moduladoras, antiinflamatorias y antiangiogénicas, relacionadas con una acción moduladora de citoquinas. ${ }^{2,4,11}$

Las propiedades antiangiogénicas se vinculan con la dismorgénesis. Varios grupos de investigadores proponen teorías directamente relacionadas con la habilidad del medicamento de inducir muerte celular y generar especies reactivas de oxígeno (ROS-reactive oxygen species), por la unión directa de la talidomida e inhibición del cereblon (CRBN), que es un componente del complejo ubiquitina ligasa., ${ }^{2,12-14}$

\section{Análogos de la talidomida}

Los análogos de la talidomida, denominados drogas inmunomoduladoras (IMiDs), son una clase de agentes desarrollados para tomar ventajas de las propiedades de la talidomida. Los nuevos análogos de la talidomida se encuentran en ensayos clínicos de fase III. ${ }^{15}$ Su desarrollo fue facilitado para una mejor comprensión de la biología en el mieloma (MM) e iniciaron un cambio profundo en el enfoque terapéutico de esta patología. ${ }^{16}$

Para aumentar las capacidades inmunomoduladoras, y por 10 tanto la actividad antitumoral de los análogos de la talidomida, se formuló la lenalidomida. ${ }^{16,17}$ La pomalidomida es la molécula IMiD más reciente y potente, y está indicada para recaídas y casos refractarios de MM. ${ }^{16}$

\section{Toxicidad}

Los efectos teratógenos de la talidomida son bien conocidos; se estima que el riesgo en seres humanos es del 10 al 5 \% con la exposición durante la gestación. ${ }^{1}$ La neuropatía periférica se presenta en el 20 - 50 \% de los pacientes. Se trata de una polineuropatía de predominio sensorial. Otros efectos indeseables de la talidomida son la sedación, presente en casi todos los pacientes, el estreñimiento, los edemas y complicaciones trombóticas. ${ }^{1,10}$

La lenalidomida, a pesar de ser un IMiDs, tiene un perfil de toxicidad diferente al de la talidomida. ${ }^{15}$ En la práctica clínica, la lenalidomida se usa generalmente en combinación con dexametasona, y los eventos tóxicos más frecuentes de esta terapia combinada incluyen: mielosupresión, infecciones y trombosis. ${ }^{15,17,18}$ El potencial papel de las modificaciones postraduccionales de CRBN o sus sustratos en la actividad de lenalidomida aún no se han determinado. ${ }^{17}$

La pomalidomida, otro análogo de la talidomida aprobado por la agencia de administradora de drogas y alimentos de los EEUU (FDA), fue teratógeno tanto en ratas como en conejos, cuando se administró durante el período de organogénesis. ${ }^{15}$ No obstante, estudios en embriones de pollo en dosis altas, no han demostrado efectos teratogénicos. ${ }^{19}$ Las complicaciones primarias reportadas por la bibliografía, en orden de aparición, son: neutropenia, anemia y trombocitopenia. ${ }^{15,20}$

\section{Regulaciones internacionales y nacionales}

La catástrofe de la talidomida en los años setenta cambió la visión acerca de los riesgos asociados a la toma de medicamentos de uso humano. Se establecieron protocolos de actuación que priorizaron la protección de la población frente a los conflictos de intereses de médicos, políticos e industria farmacéutica. ${ }^{3}$

En 1975, la FDA elaboró la clasificación de medicamentos para su uso en el embarazo, en cinco categorías de riesgo establecido por orden creciente: A, B, C, D, o X, de acuerdo con la capacidad de teratogenicidad en función del tipo de estudios realizados. ${ }^{3}$ La talidomida y sus derivados bajo esta clasificación son categoría X. ${ }^{21}$ Según la definición de la FDA, un fármaco categoría $X$ es aquel que en estudios en animales o humanos, ha demostrado anomalías fetales, o la existencia de evidencia positiva de daño fetal en humanos, y cuyo riesgo supera claramente los posibles beneficios.

En 2010, el sistema del Advisory Committee on Prescription Medicines (ACPM) reemplazó al Australian Drug Evaluation Committee (ADEC). ${ }^{22}$ El sistema del ACPM está integrado por 7 categorías (A, B1, B2, B3, C, D y X), y aunque es similar a la clasificación del FDA, difiere en ciertos aspectos, como la falta de jerarquía en su estructura. Específicamente, la categoría X se refiere a fármacos que, debido al alto riesgo de daño permanente en el feto, o a la posibilidad de este, no deben utilizarse durante el embarazo. ${ }^{22}$

Con base en el principio de precaución, debe considerarse la contracepción femenina y masculina para la categoría X, lo que disminuye el riesgo de un posible embarazo. La contracepción masculina ante la exposición con talidomida y análogos no debe obviarse, ya que la bibliografía científica disponible señala la detección en líquido seminal. ${ }^{23-25}$ 


\section{Talidomida en Costa Rica / Rivera-Chavarría, Espinoza-Aguirre}

Los Estados Unidos y el Brasil lideran programas de seguridad con el objetivo de prevenir el efecto secundario más temido: la teratogénesis.

Regulación en los Estados Unidos (Programa S. T. E. P. SSystem for Thalidomide Education and Prescribing Safety):

En 1975, la FDA aprobó el uso de la talidomida para el tratamiento de la Enfermedad de Hansen bajo ciertas condiciones. A partir de ello, muchas subespecialidades médicas solicitaron el permiso respectivo a la FDA para utilizar la talidomida, por lo que la FDA conformó el grupo de trabajo de la talidomida, en $1994 .{ }^{26}$

La FDA permitió la comercialización de la talidomida y sus análogos por Celgene, una compañía farmacéutica, en 1998. ${ }^{27}$ En conjunto, Celgene y la FDA desarrollaron el Sistema para la Educación y Prescripción Segura de la Talidomida (S.T.E.P.S- System for Thalidomide Education and Prescribing Safety). ${ }^{27}$ Este programa requiere un registro de los médicos que prescriben talidomida, como también de sus pacientes. El programa insiste en el número de medidas contraceptivas, una prueba de embarazo con resultados negativos antes de iniciar el tratamiento, prueba de embarazo al finalizar el tratamiento, y pruebas de que el paciente ha utilizado dos formas de contracepción, así como el sometimiento a pruebas de embarazo mensual durante el tratamiento. ${ }^{26}$

\section{Regulación en E1 Brasil (Ley talidomida):}

Ante el surgimiento de nuevas indicaciones terapéuticas, en 1997, el Ministro de Salud del Brasil, implementó nuevas regulaciones para un control estricto en la producción y la prescripción de la talidomida. Se prohibió la prescripción de la talidomida en mujeres en edad reproductiva, excepto para circunstancias muy especiales y bajo condiciones estrictamente controladas. ${ }^{28}$ Luego, en 2003, el Congreso Brasileño aprobó la Ley Federal No. 10.651, la Ley Talidomida, en la que prohíbe la venta comercial del medicamento y establece que este solo puede ser dispensado mediante programas avalados por el Ministerio de Salud del país. ${ }^{28}$

\section{Regulación en Costa Rica}

La talidomida no se comercializa en Costa Rica y no se encuentra registrada en el Ministerio de Salud, lo que implica que no hay proveedores que la tengan disponible para su venta en plaza. Ha ingresado al país amparada al artículo 117 de la Ley General de Salud. El artículo establece que el Ministerio de Salud, la Caja Costarricense de Seguro Social (CCSS) y cualquier otra entidad estatal, con funciones de salud pública o seguridad social, podrán adquirir medicamentos no registrados, en cualquier momento o circunstancia. En caso de urgencia o de necesidad pública, ese Ministerio podrá autorizar la importación de medicamentos no registrados. Para fines exclusivos de investigación, podrá autorizarse la importación, producción y uso de medicamentos no registrados, de conformidad con las disposiciones reglamentarias correspondientes. (Así reformado por el artículo 7 de la ley No. 6577 de 6 de mayo de1981).
El Sistema Nacional de Farmacovigilancia está regulado por medio del "Reglamento del Sistema Nacional de Farmacovigilancia"(Decreto Ejecutivo $\mathrm{N}^{\circ}$ 35244-S, Diario Oficial La Gaceta N 98, 22 de mayo de 2009), el cual aplica a la farmacovigilancia de todos los medicamentos de uso humano que se importan, fabrican, comercializan y utilizan en el país.

El Centro Nacional de Farmacovigilancia es coordinador del Sistema Nacional de Farmacovigilancia, y promotor de las Buenas Prácticas en Farmacovigilancia. De acuerdo con el Decreto Ejecutivo No35244-S, todo profesional de la salud debe notificar cualquier sospecha de reacción adversa de la que tenga conocimiento durante su práctica habitual. Establece que se debe prestar especial atención a las reacciones adversas producidas por medicamentos extranjeros no registrados, pero autorizados por el Ministerio de Salud mediante el artículo 117.

Cabe mencionar que, concomitantemente, están vigentes el Reglamento de Buenas Prácticas de Farmacovigilancia (Decreto Ejecutivo No 39417-S, Diario Oficial La Gaceta 19, 28 de enero 2016), y el Reglamento para la autorización para la importación y adquisición de medicamentos no registrados (Decreto Ejecutivo No 36358-S, Diario Oficial La Gaceta 25, 04 de febrero de 2011).

En 1982, la CCSS instituye la Lista oficial de Medicamentos (LOM) y la Normativa Institucional para la Prescripción de Medicamentos (Decreto Ejecutivo 13878-SPPS, Diario Oficial La Gaceta 25, 22 de septiembre de 1982). La LOM y la Normativa funcionan como un reglamento de acatamiento obligatorio, aprobado mediante decreto ejecutivo, y describe procedimientos de solicitud y compra de fármacos, así como indicaciones terapéuticas para su prescripción (Decreto Ejecutivo N 19343-S, Diario Oficial La Gaceta 239, 19 de diciembre de 1989). La LOM también indica los procesos de inclusión y exclusión de medicamentos a la lista, tarea que le corresponde Comité Central de Farmacoterapia de la Caja Costarricense de Seguro Social.

La LOM incluye la talidomida para uso exclusivo en dermatología, hematología, oncología, e inmunología para tratamiento de patologías específicas que no responden a otros medicamentos LOM. ${ }^{8}$ Está contraindicada en embarazadas y se debe usar con precaución en mujeres en edad fértil. Ninguno de los medicamentos análogos de la talidomida está incluido en la LOM.

Las Normas para la Atención Integral y Control de la Enfermedad de Hansen en Costa Rica (Decreto Ejecutivo ${ }^{\circ}$ 37909-S, Diario Oficial La Gaceta 204, 23 de octubre de 2013), avalan el uso de la talidomida como un medicamento de segunda línea en el tratamiento de la Enfermedad de Hansen. A pesar de que la Organización Mundial de la Salud (OMS) no recomienda su indicación como alternativa de tratamiento para el eritema nodoso leproso (ENL). ${ }^{29}$ La OMS llega a esta conclusión basada en el hecho de que el ENL no es una complicación seria, y considera más riesgoso, que la indicación del uso de talidomida como alternativa terapéutica, extienda su utilización en lugares donde no pueda ser efectivamente controlada. ${ }^{29 \cdot 31}$ 
En jurisprudencia nacional, en años recientes, destacan dos recursos presentados a la Sala Constitucional. El recurso de amparo, expediente 11-005737-0007CO Res. N ${ }^{\circ} 2011006832$, con fecha de 27 de mayo de 2011, en donde se declara con lugar un recurso contra el Hospital México en favor de la indicación de la talidomida para el tratamiento del MM, al considerar que se lesiona el derecho a la salud, por retardar la entrega del medicamento requerido para tratar el padecimiento en mención.

En segundo lugar, el 10 de octubre de 2017, la Sala Constitucional emite la sentencia $\mathrm{N}^{\circ} 16123$, Expediente 17 04908-0007, Res N²01716123, donde un paciente interpone un recurso para la sustitución del tratamiento en MM de talidomida por lenalidomida. Sin embargo, este recurso fue rechazado.

\section{Aspectos éticos}

El resurgimiento de la talidomida como tratamiento para diversas patologías arrastra una serie de consideraciones por tener presentes para su uso seguro y equilibrado. Aunque existen regulaciones dirigidas para evitar los efectos secundarios graves, que tuvo su uso en el pasado, se debe considerar que el sistema de salud costarricense lo utiliza. En vista de que hay normativas de seguridad exitosas en otros países, sería conveniente comparar y sumar esas medidas a nivel nacional.

\section{Conclusiones}

El tratamiento con talidomida y sus análogos conlleva una serie de consideraciones que se deben tomar en cuenta para su uso seguro y equilibrio riesgo / beneficio. A nivel nacional existen regulaciones tendientes a evitar los efectos secundarios graves. No obstante, debe valorarse la necesidad de actualización e implementación de nuevos sistemas de farmacovigilancia capaces de responder a las nuevas tendencias de uso.

\section{Referencias}

1. Ordi-Ros J, Cosiglio FJ. Indicaciones terapéuticas actuales de la talidomida y la lenalidomida. Med Clin (Barc). 2014; 142:360-4.

2. Vargesson N. Thalidomide-induced teratogenesis: History and mechanisms. Birth Defects Res C Embryo Today. 2015; 105:140-56.

3. Papaseit E, García-Algar O, Farré M. Talidomida: una historia inacabada. An Pediatr (Barc). 2013; 78:283-7.

4. Rehman W, Arfons LM, Lazarus HM. The Rise, Fall and Subsequent Triumph of Thalidomide: Lessons Learned in Drug Development. Ther Adv Hematol. 2011; 2:291-308

5. Kim JH, Scialli AR. Thalidomide: The Tragedy of Birth Defects and the Effective Treatment of Disease. Toxicol Sci. 2011; 122:1-6.

6. Shortt J, Hsu AK, Johnstone RW. Thalidomide-analogue biology: immunological, molecular and epigenetic targets in cancer therapy. Oncogene. 2013; 32:4191202.

7. Deng L, Ding W, Granstein RD. Thalidomide Inhibits Tumor Necrosis Factor- $\alpha$ Production and Antigen Presentation by Langerhans Cells. J Invest Dermatol. 2003; 121:1060-5.
8. CCSS. Lista Oficial de Medicamentos 2018. In: Farmacoterapia Dd, editor. San José: Caja Costarricense de Seguro Social; 2018.

9. Keifer JA, Guttridge DC, Ashburner BP, Baldwin AS, Jr. Inhibition of NF-kappa B activity by thalidomide through suppression of IkappaB kinase activity. J Biol Chem. 2001; 276:22382-7.

10. Zhou S, Wang F, Hsieh T-C, Wu JM, Wu E. Thalidomide-a notorious sedative to a wonder anticancer drug. Curr Med Chem. 2013; 20:4102-8.

11. Millrine D, Kishimoto T. A Brighter Side to Thalidomide: Its Potential Use in Immunological Disorders. Trends Mol Med. 2017; 23:348-61.

12. Ito T, Ando H, Suzuki T, Ogura T, Hotta K, Imamura Y, et al. Identification of a primary target of thalidomide teratogenicity. Science. 2010; 327:1345-50.

13. Stephens TD. Proposed mechanisms of action in thalidomide embryopathy. Teratology. 1988; 38:229-39.

14. Mori T, Ito T, Liu S, Ando H, Sakamoto S, Yamaguchi Y, et al. Structural basis of thalidomide enantiomer binding to cereblon. Sci Rep. 2018; 8:1294.

15. Liu T, Guo F, Zhu X, He X, Xie L. Thalidomide and its analogues: A review of the potential for immunomodulation of fibrosis diseases and opthalmopathy. Exp Ther Med. 2017; 14:5251-7.

16. Quach H, Ritchie D, Stewart AK, Neeson P, Harrison S, Smyth MJ, et al. Mechanism of action of immunomodulatory drugs (IMiDS) in multiple myeloma. Leukemia. 2010; 24:22-32.

17. Fink EC, Ebert BL. The novel mechanism of lenalidomide activity. Blood. 2015; 126:2366-9.

18. von Lilienfeld-Toal M, Hahn-Ast C, Furkert K, Hoffmann F, Naumann R, Bargou $\mathrm{R}$, et al. A systematic review of phase II trials of thalidomide/dexamethasone combination therapy in patients with relapsed or refractory multiple myeloma. Eur J Haematol. 2008; 81:247-52.

19. Beedie SL, Mahony C, Walker HM, Chau CH, Figg WD, Vargesson N. Shared mechanism of teratogenicity of anti-angiogenic drugs identified in the chicken embryo model. Sci Rep. 2016; 6:30038.

20. Clark SM, Steinbach A, Clemmons AB. Pomalidomide for the Treatment of Multiple Myeloma. J Adv Pract Oncol. 2014; 5:51-6.

21. Eltonsy S, Martin B, Ferreira E, Blais L. Systematic procedure for the classification of proven and potential teratogens for use in research. Birth Defects Res A Clin Mol Teratol. 2016; 106:285-97.

22. Gallego Úbeda M, Delgado Téllez de Cepeda L, Campos Fernández de Sevilla MdlA, Lorenzo Pinto Ad, Tutau Gómez F. Actualización del uso de fármacos durante el embarazo: categorías de riesgo. Farm Hosp. 2014; 38:364-78.

23. Chen N, Lau H, Choudhury S, Wang X, Assaf M, Laskin OL. Distribution of lenalidomide into semen of healthy men after multiple oral doses. J Clin Pharmacol. 2010; 50:767-74.

24. Li Y, Wang X, Liu L, Reyes J, Palmisano M, Zhou S. Distribution of pomalidomide into semen of healthy male subjects after multiple doses. Clin Pharmacol. 2018; 10:53-62.

25. Gordon JN, Goggin PM. Thalidomide and its derivatives: emerging from the wilderness. Postgrad Med J. 2003; 79:127.

26. Zeldis JB, Williams BA, Thomas SD, Elsayed ME. S.T.E.P.S.: a comprehensive program for controlling and monitoring access to thalidomide. Clin Ther. 1999; 21:319-30.

27. Bwire R, Freeman J, Houn F. Managing the teratogenic risk of thalidomide and lenalidomide: an industry perspective. Expert Opin Drug Saf. 2011; 10:3-8.

28. Paumgartten FJR, Souza NRd. Clinical use and control of the dispensing of thalidomide in Brasilia-Federal District, Brazil, from 2001 to 2012. Cien Saude Colet. 2013;18:3401-8

29. Crawford CL. No role for thalidomide in the treatment of leprosy. J Infect Dis 2006; 193:1743-4

30. Crawford CL. Treatment of erythema nodosum leprosum with thalidomide. Lancet. 1973; 2:567-8.

31. Haslett PAJ, Kaplan G. Reply to Crawford. J Infect Dis. 2006; 193:1744-5. 